\title{
Shallow-terrace-like interface in dilute-bismuth GaSb/AIGaSb single quantum wells evidenced by photoluminescence
}

\author{
Xiren Chen, ${ }^{1}$ Yuxin Song, ${ }^{2}$ Liang Zhu, ${ }^{1}$ S. M. Wang, ${ }^{2, a)}$ Wei Lu, ${ }^{1}$ Shaoling Guo, ${ }^{1}$ \\ and Jun Shao ${ }^{1, b)}$ \\ ${ }^{1}$ National Laboratory for Infrared Physics, Shanghai Institute of Technical Physics, \\ Chinese Academy of Sciences, 200083 Shanghai, China \\ ${ }^{2}$ Photonics Laboratory, Department of Microtechnology and Nanoscience, \\ Chalmers University of Technology, SE-412 96 Göteborg, Sweden
}

(Received 15 December 2012; accepted 27 March 2013; published online 15 April 2013)

\begin{abstract}
Photoluminescence (PL) measurements are performed on one GaSb/AlGaSb single-quantum-well (SQW) sample and two dilute-bismuth (Bi) GaSb/AlGaSb SQW samples grown at 360 and $380{ }^{\circ} \mathrm{C}$, at low temperatures and under magnetic fields. Bimodal PL features are identified in the dilute-Bi samples, and to be accompanied by abnormal PL blueshift in the sample grown at $360{ }^{\circ} \mathrm{C}$. The bimodal PL features are found to be from similar origins of band-to-band transition by magneto-PL evolution. Analysis indicates that the phenomenon can be well interpreted by the joint effect of interfacial large-lateral-scale islands and $\mathrm{Al} / \mathrm{Ga}$ interdiffusion due to $\mathrm{Bi}$ incorporation. The interdiffusion introduces about 1-monolayer shrinkage to the effective quantum-well thickness, which is similar to the interfacial islands height, and the both together result in an unusual shallow-terracelike interface between GaSbBi and AlGaSb. A phenomenological model is established, the Bi content of isoelectronic incorporation and the exciton reduced effective mass are estimated for the $\mathrm{GaSbBi}$ sample grown at $380^{\circ} \mathrm{C}$, and a value of about $21 \mathrm{meV} / \%$ is suggested for the bandgap bowing rate of $\mathrm{GaSbBi}$. An effective routine is suggested for determining the Bi content and the depth of the
\end{abstract} shallow-terraces at interface in dilute-Bi SQW structures. (C) 2013 AIP Publishing LLC

[http://dx.doi.org/10.1063/1.4801530]

\section{INTRODUCTION}

Dilute bismuth (Bi) III-V compound semiconductors have recently attracted great attention because of the potential applications in near infrared emitters and detectors, ${ }^{1}$ among which $\mathrm{GaAsBi}$ is of current interest mainly due to its large band-gap reduction, ${ }^{1,2} \mathrm{Bi}$-induced temperatureinsensitive emission wavelength, ${ }^{3}$ and giant spin-orbit bowing. ${ }^{4}$ In comparison to $\mathrm{GaAsBi}, \mathrm{GaSbBi}$ is expected to manifest further advantages of efficient photoelectronic devices for the $1.55 \mu \mathrm{m}$ optical communication window and easy realization of suppressing Auger recombination. ${ }^{5}$ Similar to dilute nitrogen $(\mathrm{N})$ compound semiconductors, the band gap of dilute-Bi semiconductor is expected to shrink relative to the non-Bi host semiconductor. However, difference is also distinct that the electronic structure effects of $\mathrm{Bi}$ incorporation, e.g., band anticrossing (BAC) ${ }^{6}$ manifest mainly on valence band ${ }^{7}$ rather than conduction band, ${ }^{8}$ which may offer another choice for semiconductor electronic band engineering and optoelectronic applications. It is unfortunate, however, that to date experimental study of GaSbBi was very limited, ${ }^{9-11}$ and the situation was even worse for optical investigation of low-dimensional heterostructures because of the difficulty of Bi incorporation.

\footnotetext{
${ }^{a)}$ Electronic mail: shumin@chalmers.se

b) Author to whom correspondence should be addressed. Electronic mail: jshao@mail.sitp.ac.cn
}

Photoluminescence (PL) as nondestructive optical spectroscopy has found wide applications in characterizing electronic structures and optical properties of semiconductors, ${ }^{12-15}$ especially for nanometer-thick thin layers of which alloy content is difficult to be determined by, e.g., $\mathrm{x}$-ray diffraction (XRD) and secondary ion mass spectroscopy (SIMS). PL has been also used to study the quality and the morphology of the heterostructure interface for low dimensional semiconductor system such as quantum well or superlattice. ${ }^{16-20}$ Interfacial atom interdiffusion and physical morphology affect the electronic structure, and lead energy shift, inhomogeneous broadening, and intensity degradation to the PL feature of band-to-band transition. Therefore, quantitative PL lineshape analysis of bandedge-related transitions can serve as an effective pathway for acquiring heterointerfacial information. Meanwhile, magneto-PL is useful for clarifying excitonic effect and possible origin of particular transitions, and has found wide applications in the lowdimensional semiconductors. ${ }^{21,22}$ For the materials with strong bandtail states like dilute-N, however, accurate information may be drawn only when the real band-to-band transition is discriminated from defects-induced processes, which is unfortunately not straightforward.

In this study, PL and magneto-PL measurements are carried out at low temperatures on $\mathrm{GaSb}(\mathrm{Bi}) / \mathrm{AlGaSb}$ single quantum well (SQW) samples grown at different temperatures. Bimodal PL features are observed, and abnormal blueshift is identified for the sample grown at $360^{\circ} \mathrm{C}$ while redshift is obvious for the sample grown at $380^{\circ} \mathrm{C}$. The 
results suggest that (i) Bi-induced shallow-terrace-like interface is responsible for the bimodal PL features, which is a joint effect of $\mathrm{Al} / \mathrm{Ga}$ interdiffusion and large-lateral-scale islands formation at the interface. (ii) The GaSbBi SQW sample grown at $380^{\circ} \mathrm{C}$ has higher isoelectronic-incorporated $\mathrm{Bi}$ content, and the incorporation of $\mathrm{Bi}$ enhances the exciton reduced effective mass and binding energy.

\section{EXPERIMENTAL DETAILS}

Two $\mathrm{GaSb}_{1-x} \mathrm{Bi}_{x} / \mathrm{AlGaSb}$ and one GaSb/AlGaSb SQW samples used in this study were grown on $\mathrm{GaSb}$ substrate at 360,380 , and $370^{\circ} \mathrm{C}$ (denoted by B360, B380, and R370 hereafter), respectively, by molecular beam epitaxy (MBE) with a structure of $5-\mathrm{nm}-\mathrm{GaSb}(\mathrm{Bi})$ well sandwiched by $100-\mathrm{nm}-\mathrm{Al}_{0.2} \mathrm{Ga}_{0.8} \mathrm{Sb}$ barriers and a $10-\mathrm{nm}-\mathrm{GaSb}$ capping layer. Since Bi content of such thin SQW was difficult to be determined by XRD or SIMS, it was preliminarily assumed to be $x \simeq 0.2 \%$ and $0.7 \%$, respectively, for the B360 and B380 samples, by the XRD rocking curves and the SIMS of the GaSbBi thin films grown by the same MBE system. ${ }^{5}$ For the speculation of possible interfacial ununiformity of the samples, 200-nm-thick $\mathrm{GaSb}(\mathrm{Bi})$ epilayers were prepared by the same MBE system at nominally identical temperatures as the samples B360, R370, and B380, respectively, and surface morphologic characterization was conducted by atomic force microscopic (AFM) technique. ${ }^{5}$

PL measurements were conducted with a spectral resolution of $1 \mathrm{meV}$ (or equivalently, $8 \mathrm{~cm}^{-1}$ ) using a Fourier transform infrared (FTIR) spectrometer (Bruker IFS 66v/S) in the rapid- rather than step-scan mode. ${ }^{13,22} \mathrm{Ar}^{+}$laser at 514.5-nm line was used together with a laser power controller to warrant a stable excitation at $60 \mathrm{~mW}$. The luminescence signal was detected by a room temperature InGaAs detector. The samples were cooled down either by liquid nitrogen to about $77 \mathrm{~K}$ or by continuous flow liquid helium to about 3.8 K. Detailed description of the PL system and experimental configuration can be found elsewhere. ${ }^{13,20}$ Magnetic fields of up to $10 \mathrm{~T}$ were built up using a superconducting optical magnet in Faraday configuration. ${ }^{21,22}$ The spatial distance between the FTIR spectrometer and the optical magnet was far enough to guarantee the elimination of the interference on the instrument performance by magnetic field.

\section{RESULTS AND DISCUSSION}

Figure 1(a) illustrates a PL spectrum and its inverted second-order derivative (SOD) of the $\mathrm{R} 370 \mathrm{GaSb} / \mathrm{AlGaSb}$ SQW sample at $77 \mathrm{~K}$.

The slightly asymmetric single-peak SOD lineshape hints a dominant PL process accompanied by a close-positioned high-energy weak PL process, and the peak corresponds to the energy of the dominant PL process. The transition energy of the dominant PL process is determined ${ }^{22}$ as $0.9229 \mathrm{eV}$. To account for the asymmetry of the PL lineshape, gravity-center (GC) energy is also estimated as $0.9249 \mathrm{eV}$ by taking the total PL transitions as a single visual process, the position is marked by the vertical bar in Fig. 1(a). Similarly, the dominant PL and the GC energies are determined as 0.9310 and $0.9288 \mathrm{eV}$ for the $\mathrm{B} 360$, and 0.9194 and $0.9172 \mathrm{eV}$ for the

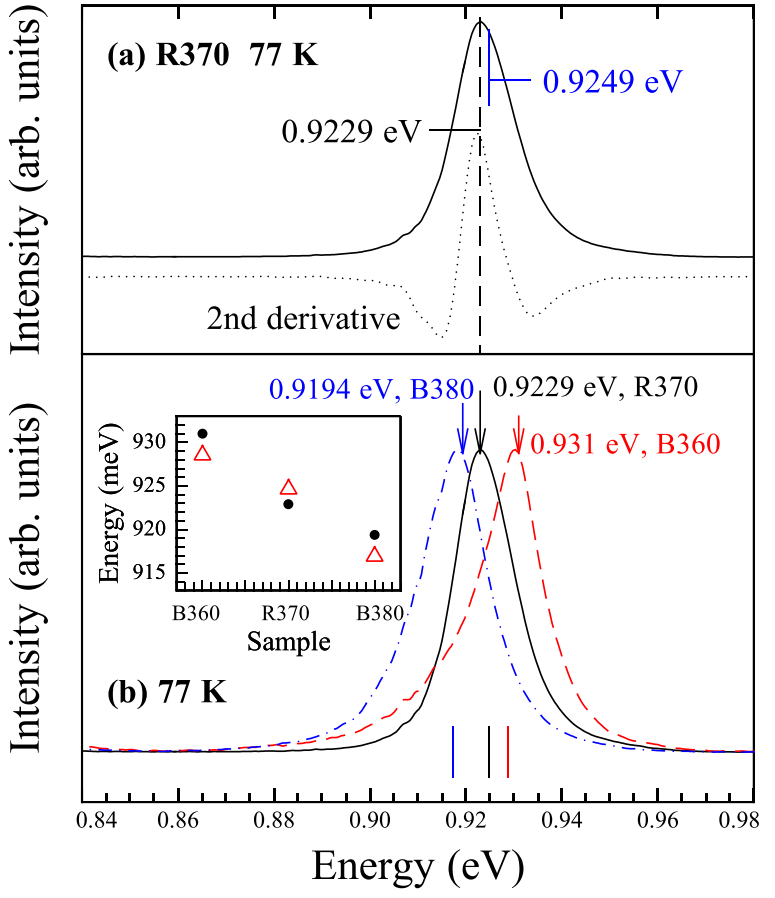

FIG. 1. (a) PL spectrum and its inverted second derivative of R370 GaSb/ AlGaSb SQW sample at $77 \mathrm{~K}$, vertical dashes for peak position and vertical bar for GC energy. (b) Normalized PL spectra of B360 and B380 GaSbBi/ $\mathrm{AlGaSb}$ and R370 GaSb/AlGaSb SQW samples. Peak energies by vertical arrows and digits, and GC energies by bars at the bottom. Inset shows peak and corresponding GC energies (dots and open triangles).

B380 GaSbBi/AlGaSb SQW samples at $77 \mathrm{~K}$, the results are indicated by the vertical arrows and bars in Fig. 1(b) together with that of the R370 GaSb sample. The difference of the dominant PL and GC energies is plotted as inset in Fig. 1(b) for all the three samples. The dots represent the peak energies, while the open triangles the GC energies.

The PL spectra of the two dilute-Bi samples manifest typical features as widely observed in dilute- $\mathrm{N}$ materials, ${ }^{23,24}$ i.e., one dominant PL process of band-to-band transition and a close-positioned weak bandtail of defect-/ interfacial roughness-related localization. The PL peak of $\mathrm{B} 380$ redshifts relative to $\mathrm{R} 370$, due to the $\mathrm{Bi}$ isoelectronic incorporation-induced valence-band shifting and bandgap shrinking as predicted by the BAC theory. It is strange, however, that B360 manifests an abnormal blueshift of the PL peak relative to R370, which is difficult to understand solely with the BAC model.

As the linewidth of PL feature is determined by homogeneous and inhomogeneous broadening, of which the former gets enhanced at higher temperatures when phonon scattering comes into force, ${ }^{25}$ similar PL measurements are performed at a low temperature of $3.8 \mathrm{~K}$ to diminish the homogeneous broadening. The results are shown in Fig. 2. The peak energy is again determined by the SOD as $0.9307 \mathrm{eV}$ for R370. For the GaSbBi samples, however, two features are identified with energies of 0.9309 and $0.9385 \mathrm{eV}$ for B360, and 0.9202 and $0.9263 \mathrm{eV}$ for B380. The GC energies are also estimated as $0.9288,0.9305$, and $0.9214 \mathrm{eV}$, respectively, for the samples R370, B360, and B380. The difference of the dominant $\mathrm{PL}$ and GC energies is plotted as inset in Fig. 2 for all the 


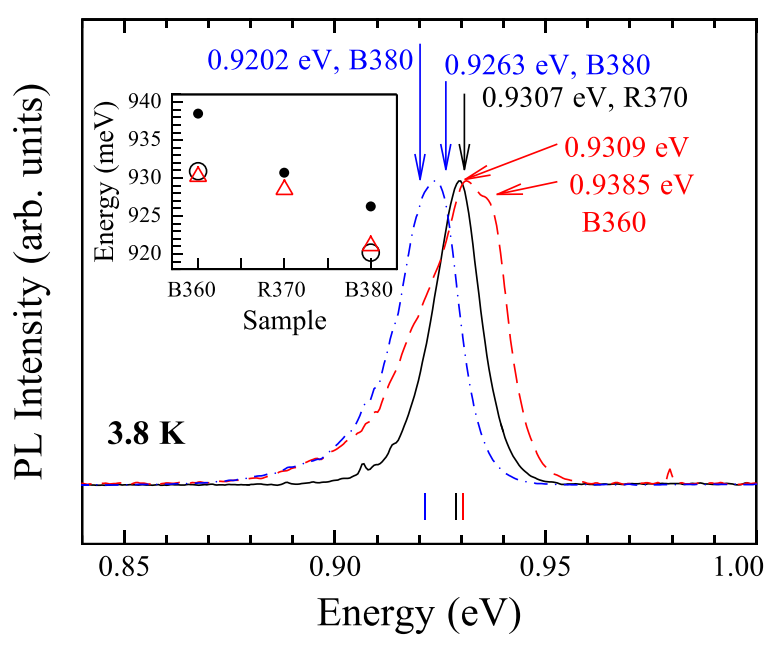

FIG. 2. Normalized PL spectra of the samples R370, B360, and B380 at 3.8 K. 2nd-derivative energies marked by arrows and digits, and GC energies by vertical bars. Inset: 2nd-derivative energies as dots and open circles, and GC energies as open triangles, respectively.

three samples. The dots and open circles represent the SOD energies, and the open triangles show the GC energies.

The PL spectrum of R370 manifests a similar lineshape as that at $77 \mathrm{~K}$, and the difference of the $\mathrm{GC}$ energies between 3.8 and $77 \mathrm{~K}$ is $3.9 \mathrm{meV}$. For the GaSbBi samples, however, bimodal rather than unimodal features are identifiable with an energetic separation of $7.6 \mathrm{meV}$ for $\mathrm{B} 360$ and $6.1 \mathrm{meV}$ for B380. The difference of the GC energies between 3.8 and $77 \mathrm{~K}$ is $4.2 \mathrm{meV}$ for $\mathrm{B} 380$, close to that of R370, but $1.7 \mathrm{meV}$ for B360, which is much smaller than that of the other two samples. The energy of the lower-energy PL feature of B360 (as indicated by open circle in the inset of Fig. 2) is nearly identical to the energy of the dominant peak of R370 (as indicated by dot in the inset). The abnormal blueshift of B360 at $77 \mathrm{~K}$ seems to be the effect of carrier population in a higherenergy state, of which the transition energy is $0.9385 \mathrm{eV}$ at $3.8 \mathrm{~K}$ and the origin will be clarified later when a phenomenological model is devised for Bi effect. Homogeneous broadening merges the PL features to a broader peak, while the difference of GC energy between 3.8 and $77 \mathrm{~K}$ is a hint for carrier population in higher-energy state.

To clarify the mechanisms of the PL features and to verify if they reflect excitonic effects, magneto-PL measurements are conducted at $3.8 \mathrm{~K}$ under Faraday configuration ${ }^{21,22}$ at various magnetic fields up to $10 \mathrm{~T}$. Representative spectra are depicted in Fig. 3. Curve fittings with a mixture of Lorentzian and Gaussian (MLG) functions are performed, ${ }^{20,25}$ the results are plotted also in Fig. 3 as dots, dashes, and dashed-dots, respectively.

The PL spectra of R370 are as illustrated in Fig. 3(a) well reproduced by a dominant feature (denoted by DF) centered at $0.9302 \mathrm{eV}$ at $0 \mathrm{~T}$ and a defects and/or interfacial roughness-induced localized states-related bandtail, which is of typical PL characteristic of QW structures. ${ }^{25}$ The former should originate from the band-to-band transition. It is worthy to mention that the DF energy at $0 \mathrm{~T}$ is nearly identical to that derived by the SOD, indicating the reliability of the MLG fittings. For B360 and B380, on the other hand, four MLGs are needed in reproducing the magneto-PL spectra as illustrated in Figs. 3(b) and 3(c), of which the two PL features at high energy side correspond to the bimodal features identified in Fig. 2 and are denoted by low-energy feature (LEF) and high-energy feature (HEF), the third is broad and strong feature denoted by BSF, and the fourth with the lowest energy is of bandtail characteristic just as that of R370. The energies of LEF and HEF are 0.9312 and $0.9381 \mathrm{eV}$ for $\mathrm{B} 360$, and 0.9205 and $0.9266 \mathrm{eV}$ for $\mathrm{B} 380$ at $0 \mathrm{~T}$. The ratio of the LEF and HEF integral intensities is about $33.2 \%$ and $48.3 \%$, respectively, for B360 and B380, indicating the population of the higher-energy state to be relatively higher for B360. This is in good agreement with the above judgment from the difference of the $\mathrm{GC}$ energies at 3.8 and $77 \mathrm{~K}$.

For the BSF in the samples B360 and B380, correlation to inhomogeneous broadening can be assumed based on the facts that: (i) the BSF only shows up in the GaSbBi samples, (ii) its energy is lower than that of the LEF and HEF, and (iii) it is only of Gaussian lineshape in the whole magnetic fields of $0-10 \mathrm{~T}$. As Bi is known to create bound states and disorder of the structure matrix, ${ }^{2,26}$ the BSF can thus be assigned to the structure disorder-related transitions in the GaSbBi samples. For the LEF and HEF, the mechanism seems to be unlikely the inhomogeneous Bi incorporation simply due to the PL blueshift of B360 relative to R370. In contrast, these features may directly correlate to the band-to-band transitions in different parts of the QW layer with considerable difference in thickness introduced by interfacial large-lateral-scale islands. Recent AFM experiment indicated that the incorporation of $\mathrm{Bi}$ in $\mathrm{GaSb}$ thin film may result in regular step-like terraces or islands at the surface with a lateral scale of $\sim 10^{2} \mathrm{~nm}$ and thickness of $\sim 10^{-1}-10^{0} \mathrm{~nm}$, which is more significant than the GaSb reference sample. ${ }^{5}$ Meanwhile, a thickness difference of 1 monolayer (ML, or about $0.3 \mathrm{~nm}$ ) in 5-nm-thick $\mathrm{GaSb} / \mathrm{Al}_{0.2} \mathrm{Ga}_{0.8} \mathrm{Sb} \mathrm{SQW}$ will lead to about $6 \mathrm{meV}$ change to the band-to-band transition energy, according to the effective-mass approach for finite-depth $\mathrm{QW}^{27}$ with an $80-\mathrm{meV}$ valence-band offset $^{28}$ and without nonparabolic effects. As the excitation laser spot is at a size of $10^{2} \mu \mathrm{m}$ and especially, the energy difference between LEF and HEF is about $7 \mathrm{meV}$ for B360 and $6 \mathrm{meV}$ for B380, the bimodal PL features of LEF and HEF are likely a sign of the formation of

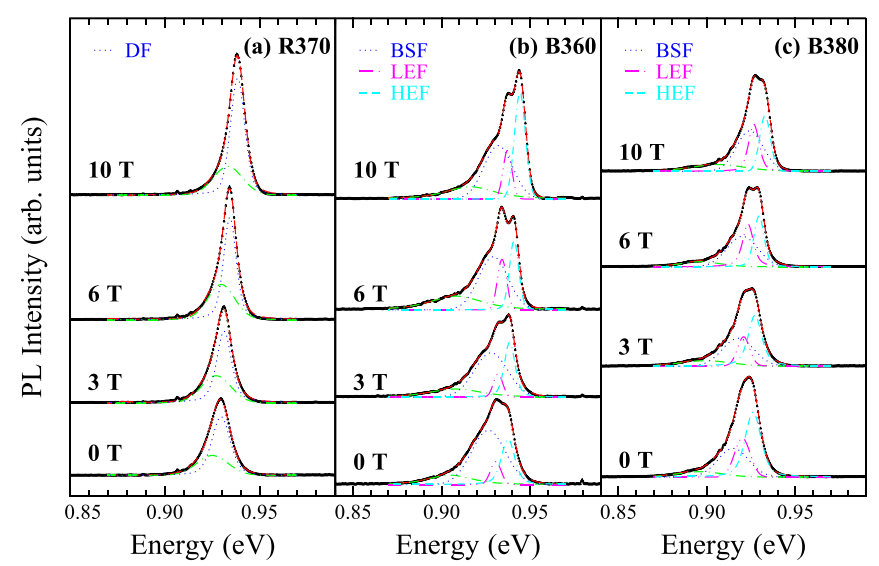

FIG. 3. Representative magneto-PL spectra and curve fittings of R370 (a) $\mathrm{GaSb} / \mathrm{AlGaSb}$, and B360 (b) and B380 (c) GaSbBi/AlGaSb SQW samples at $3.8 \mathrm{~K}$. Dots in (a) for DF of R370. Dots, dashed-dots, and dashes in (b) and (c) for the curve fittings of BSF, LEF, and HEF of B360 and B380, respectively. 
terraces or islands across the interface between the $\mathrm{GaSbBi}$ well and the AlGaSb barrier layers. ${ }^{5}$

It is worthy to mention that bimodal PL features were also observed in GaAs/AlAs quantum wells. ${ }^{16,18}$ However, there is a significant difference between the bimodal PL of $\mathrm{GaAs} / \mathrm{AlAs}$ QWs ${ }^{16,18}$ and that of GaSbBi SQW in this study. The interfacial islands result in PL redshift for the GaAs/ AlAs QWs because of the low-energy-state occupation of carriers at low temperature, while abnormal blueshift in B360 GaSbBi SQW sample is observed in this study, indicating different mechanism for the bimodal PL in GaAs/AlAs and GaSbBi QW systems.

Figure 4 plots the energies of the DF, LEF, and HEF, respectively, as squares, open stars, and dots, against magnetic field for the three samples. The LEF and HEF of B360 are upshifted by $5 \mathrm{meV}$ for clarity. Clearly, all the features manifest typical diamagnetic shift, indicating the nature of excitonic transition. ${ }^{21}$ The BSF and the bandtail feature in Fig. 3, on the other hand, cannot be assumed with diamagnetic shift because of strong fluctuation of the energies around a baseline.

The evolutions of the features' energies with magnetic field are fitted as solid lines by a quasi-two-dimensional exciton effective-mass equation ${ }^{21,22}$

$$
\begin{gathered}
\left\{-\frac{\hbar^{2}}{2 \mu}\left[\frac{1}{\rho} \frac{\partial}{\partial \rho}\left(\rho \frac{\partial}{\partial \rho}\right)\right]+\frac{e^{2}}{8 \mu} B^{2} \rho^{2}-V(\rho)\right\} R_{n}(\rho)=E_{n} R_{n}(\rho), \\
V(\rho)=\frac{e^{2}}{4 \pi \varepsilon_{0}} \int_{-\infty}^{+\infty} \frac{\left|Z_{e}\left(z_{e}\right)\right|^{2}\left|Z_{h}\left(z_{h}\right)\right|^{2}}{\varepsilon_{r}\left(z_{e}, z_{h}\right) \sqrt{\rho^{2}+\left(z_{e}-z_{h}\right)^{2}}} d z_{e} d z_{h}
\end{gathered}
$$

where $\mu$ is the exciton reduced effective mass. $\rho$ is the extent of the excitonic in-well-plane wave function. $Z_{e}\left(z_{e}\right)$ and $Z_{h}\left(z_{h}\right)$ are the wave functions of electrons and holes in confined direction, respectively. $\epsilon_{0}$ and $\epsilon_{r}$ are the absolute and relative permittivity of the material, respectively. The

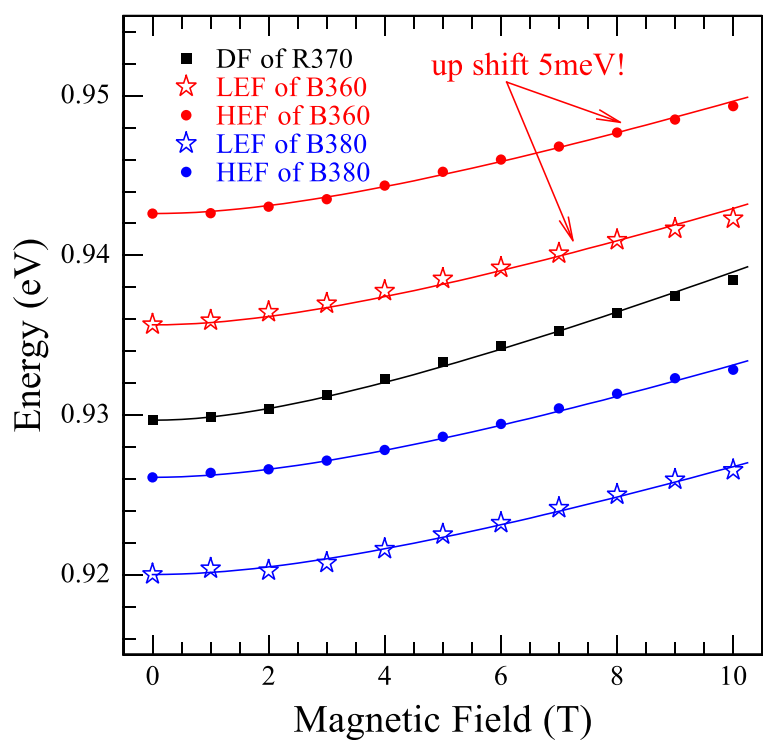

FIG. 4. PL energies of DF of R370 GaSb/AlGaSb, and LEF and HEF of $\mathrm{B} 360$ and B380 GaSbBi/AlGaSb SQW samples at $3.8 \mathrm{~K}$, respectively, as a function of magnetic field. Solid lines are the fittings with an exciton effective-mass approach. LEF and HEF of B360 are up-shifted by $5 \mathrm{meV}$ for clarification.
TABLE I. Exciton reduced effective mass $\left(\mu\right.$, in the unit of $\left.m_{0}\right)$ and binding energy $\left(E_{b}\right.$, in the unit of $\mathrm{meV}$ ) of the DF of GaSb/AlGaSb SQW sample R370, and the LEF and HEF of GaSbBi/AlGaSb SQW samples B360 and B380.

\begin{tabular}{llllllll}
\hline \hline & \multicolumn{2}{c}{ B360 } & & \multirow{2}{*}{ R370 } & & \multicolumn{2}{c}{ B380 } \\
\cline { 2 - 3 } \cline { 7 - 8 } & LEF & HEF & & DF & & LEF & HEF \\
\hline$\mu$ & 0.0365 & 0.0373 & & 0.0315 & 0.0382 & 0.0373 \\
$E_{b}$ & 7.67 & 7.79 & & 6.88 & & 7.93 & 7.79 \\
\hline \hline
\end{tabular}

subscript $n$ represents the $n$th excitonic state. $R_{n}(\rho)$ is the envelope function of $S$ symmetry exciton. The results are depicted as lines in Fig. 4. With this procedure, the exciton reduced effective mass and binding energy are derived for the DF of the sample R370, LEF and HEF of the samples B360 and B380, and are listed in Table $\mathrm{I}$ in the unit of $m_{0}$ and $\mathrm{meV}$, respectively.

It is obvious that the excitonic effects in the dilute- $\mathrm{Bi}$ samples B360 and B380 are enhanced relative to the R370 reference sample, while the difference between the two dilute-Bi samples is relatively small. In addition, the effects are similar for the LEF and HEF, with less than $2.5 \%$ difference in either reduced effective mass or exciton binding energy, and the exciton radii of the LEF and HEF are both about $20 \mathrm{~nm}$, much smaller than the lateral scale of the terraces revealed by the AFM morphology of GaSbBi thin film. ${ }^{5}$ Figure 5 shows the AFM surface morphology of an area of $3 \times 3 \mu \mathrm{m}^{2}$ of 200 -nm-thick $\mathrm{GaSb}(\mathrm{Bi})$ thin film with the growth condition as B360 (a), R370 (b), and B380 (c). It is seen in Figs. 5(a) and 5(c) that obvious large-lateral-scale islands emerge only in the dilute-Bi films. The lateral scale of the islands is estimated to be at a level of $10^{2} \mathrm{~nm}$, by which a similar size of islands can be projected to the SQW structure if the in-plane strain in the 5-nm-thick QW layer is taken as the same as the 200-nm-thick thin film. It was shown that the lateral scale of the island is mainly determined by the crystal strain, ${ }^{29}$ within the critical thickness of the thin layer. The root mean square (RMS) is about $0.294 \mathrm{~nm}, 0.185 \mathrm{~nm}$, and $0.298 \mathrm{~nm}$, respectively, for the three samples, indicating significantly rougher surface for the GaSbBi thin films. These results suggest the mechanism of the LEF and HEF processes as band-to-band transitions with different QW thicknesses. Such a difference in QW thickness is, however, different from the case as GaAs/AlAs QW ${ }^{16,18}$ with quantum islands at the interface, because of the abnormal blueshift in the GaSbBi sample B360 as mentioned above.

To well understand the experimental observation, a phenomenological model is proposed as illustrated in Fig. 6, based on the interfacial larger-lateral-scale islands and the atomic interdiffusion at the interface. Bi was recently indicated as a potential factor for increasing the III-group atomic interdiffusion in alloys. ${ }^{30}$ Thus, $\mathrm{Al} / \mathrm{Ga}$ interdiffusion across the GaSbBi/AlGaSb interface is enhanced. The energy difference between the LEF and HEF is about $7 \mathrm{meV}$ for B360, which corresponds to about 1.1-ML-thick interfacial islands induced by Bi incorporation as schematically illustrated in Fig. 6(a). On the other hand, as the LEF energy of B360 is almost equal to, and the HEF energy takes a blueshift 

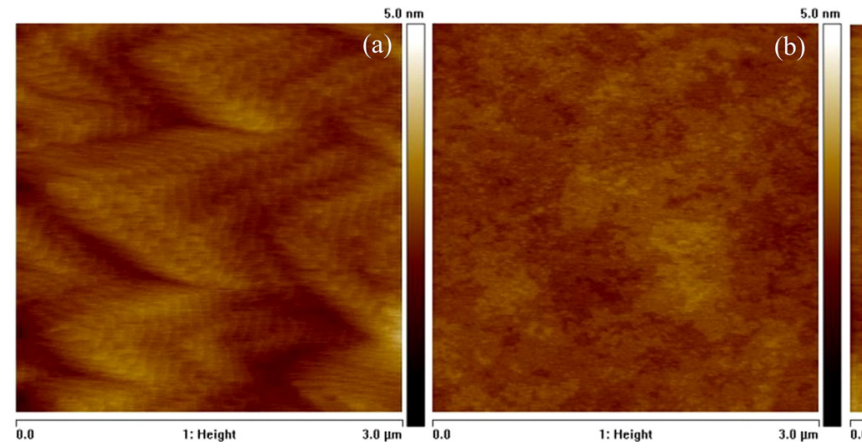

relative to the DF energy of $\mathrm{R} 370$, the isoelectronic $\mathrm{Bi}$ content in $\mathrm{B} 360$ can be negligible, and the $\mathrm{Al} / \mathrm{Ga}$ interdiffusion is equivalent to a shrinkage of $\mathrm{QW}$ thickness by $1.2 \mathrm{ML}$ according to the blueshift of HEF, as indicated in Fig. 6(b). The interdiffusion-induced shrinkage of QW thickness neutralizes the effect of the islands, and results in shallow terraces where there are initially not islands. The joint effect of the islands and the $\mathrm{Al} / \mathrm{Ga}$ interdiffusion at the interface both induced by $\mathrm{Bi}$ incorporation is as a consequence the emergence of an effective shallow-terrace-like interface structure at the GaSbBi QW side as shown in Fig. 6(c). The LEF should hence correlate to the band-to-band transition of 5nm-thick QW, while the HEF the band-to-band transition of the shallow-terrace related part of the QW, respectively. Large amount of the shallow terraces and significant thermalization of the carriers initially distributed in the LEF related states lead to a blueshift of the PL peak for the GaSbBi sample B360 at $77 \mathrm{~K}$ as aforementioned.

It is interesting to note that the formation of the interfacial large-lateral-scale islands in GaSbBi thin films is also justified by the difference in the AFM surface morphology from the GaSb thin film as illustrated in Fig. 5. While the both GaSbBi thin films grown at 360 and $380{ }^{\circ} \mathrm{C}$ show clear lateral-scale islands with a RMS of about $0.30 \mathrm{~nm}$, the $\mathrm{GaSb}$ thin film grown at $370^{\circ} \mathrm{C}$ does not show such lateral-scale islands obviously, and the RMS of the surface morphology is only about $0.19 \mathrm{~nm}$.

(a)
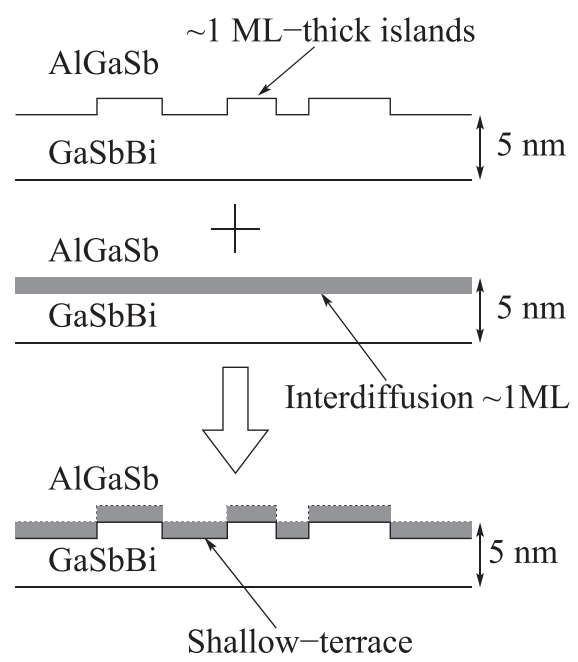

FIG. 6. Schematic for the formation of shallow-terrace-like interface (c) in $\mathrm{B} 360$ and B380 GaSbBi/AlGaSb SQW samples due to Bi-enhanced interfacial large-lateral-scale islands (a) and atomic interdiffusion (b).

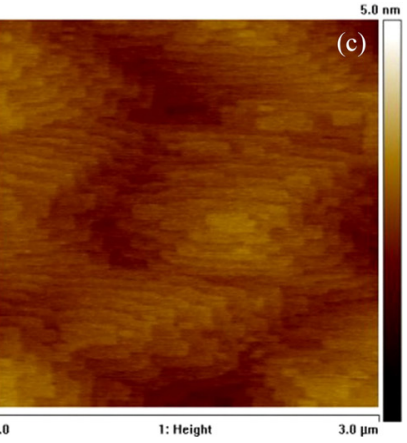

FIG. 5. AFM surface morphology of an area of $3 \times 3 \mu \mathrm{m}^{2}$ of 200 -nm-thick $\mathrm{GaSb}(\mathrm{Bi})$ thin film with the growth condition as B360 (a), R370 (b), and B380 (c), respectively, with a root mean square of about $0.294 \mathrm{~nm}, 0.185 \mathrm{~nm}$, and $0.298 \mathrm{~nm}$.

Taking into account the facts that (i) the LEF of B360 shows up at nearly the identical energetic position as, while the LEF and HEF of B380 redshift relative to the DF of $\mathrm{R} 370$, and (ii) the BSF correlating to structural disorder is more significant for B360, preliminary conclusion can be drawn that lower growth temperature generates more disorder and defects, and the Bi-induced bandgap reduction should be very small. At a higher growth temperature of $380{ }^{\circ} \mathrm{C}$, Bi tends to occupy the substitutional sites to shrink the bandgap energy by rising the topmost valence band, and the effect of the shallow-terraces is surpassed, which leads to about $10 \mathrm{meV}$ redshift at $3.8 \mathrm{~K}$. The large difference in PL intensities of the dominant band-to-band and bandtail transitions in the GaSb sample R370, on the other hand, suggests a superior interface and structure quality over the dilute-Bi counterparts.

The exciton reduced effective mass and binding energy of the LEF of B380 are about $21 \%$ and $16 \%$ larger than those of the DF of R370, respectively. This is in agreement with the established knowledge that dilute-Bi isoelectronic incorporation enlarges the reduced effective mass. ${ }^{31}$ For B360, the $\mathrm{Bi}$ isoelectronic incorporation should be very limited, the enlarged reduced effective mass should therefore not mainly be the isovalent Bi effect. Previous $\mathbf{k} \cdot \mathbf{p}$ simulation indicated that structural disorder may result in an increase to the effective mass. ${ }^{32}$ As the BSF features are also related to the structural disorder and the proportions in integral intensity are about $55 \%$ and $29 \%$, respectively, for B360 and B380 at $0 \mathrm{~T}$, the effective mass enlargement may be correlated to structural disorder in the GaSbBi sample B360 while it should be a joint effect of disorder and $\mathrm{Bi}$ isoelectronic incorporation in the GaSbBi sample B380.

The combination of the temperature and magnetic-field effects on PL properties leads to a qualitative description of $\mathrm{Bi}$ in dilute-Bi GaSb QW layer that Bi atoms exert mainly two influences on $\mathrm{GaSb} / \mathrm{AlGaSb} \mathrm{SQW}$ of (i) incorporating into the substitutional sites to form a real dilute-Bi ternary compound, and (ii) resulting in the formation of shallowterraces-like interface structure. While the former results in a bandgap reduction and exciton enhancement as clearly seen in the GaSbBi SQW sample B380, the latter forms a partial narrower QW which corresponds to higher transition energy and disorder-enhanced two-dimensional excitonic effects as evidenced in the GaSbBi SQW sample B360. Growth temperature dominates $\mathrm{Bi}$ behavior. At a growth temperature of $380{ }^{\circ} \mathrm{C}, \mathrm{Bi}$ atoms are encouraged to occupy substitutional sites, the bandgap shrinkage surpasses the shallow-terrace 
interface effect. At $360^{\circ} \mathrm{C}$, however, low-terrace effect dominates and leads to an overall PL blueshift.

Implications are as follows noteworthy for the identification of Bi effects in QWs: (i) The LEF at extremely low temperature is considered as the real band-to-band transition with the nominal QW thickness, its redshift relative to the DF of the reference GaSb sample can be used for bandgap bowing $^{1}$ analysis reliably. (ii) The energy separation as well as the relative strength of the LEF and HEF can serve as a judgment for the degree of the low-terraces depth and the distribution of the unidirectional terraces. (iii) The LEF of the sample B380 redshifts by $9.7 \mathrm{meV}$ relative to the reference sample R370. It corresponds to about $15 \mathrm{meV}$ up-shifting of the topmost valence-band edge, with the assumption that $\mathrm{Bi}$ isoelectronic doping only rises the topmost valence band within the approach of model-solid theory ${ }^{27}$

$$
\Delta E_{\text {offset }}=E_{\text {con }}\left[\frac{m_{w}^{*}}{m_{w}^{*}+m_{b}^{*} \tan ^{2}(k L / 2)}\right]^{-1 / 2},
$$

where $\Delta E_{\text {offset }}$ is the valence-band offset between the QW and the barrier layers, $E_{\mathrm{con}}$ is the confinement energy of the first confined hole level, $m_{w}^{*}$ and $m_{b}^{*}$ are the carrier effective masses in the QW and the barrier layers, respectively, $L$ is the QW width, $k$ is the wave vector given by $k=\sqrt{2 m_{w}^{*} E_{\text {con }}} / \hbar$. The bandgap bowing rate of GaSbBi is hence estimated to be about $21 \mathrm{meV} / \%$ if the Bi content is taken as $0.7 \%$ as that of thin film.

It is worthy to mention that two significantly different values of $33 \mathrm{meV} / \%^{9}$ and $100 \mathrm{meV} / \%^{11}$ were previously suggested. However, if the facts are taken into account that (i) in the BAC theory, the bandgap bowing in dilute-Bi material is due to the interaction of the resonant Bi energy level with the topmost valence-band edge of the host material, (ii) the absolute Bi energy level does not vary significantly in different matrix, ${ }^{7}$ and (iii) the topmost valence-band edge of $\mathrm{GaSb}$ is higher than that of $\mathrm{GaAs},{ }^{33}$ it is rather safe to consider that the bowing rate of GaSbBi should not be lager than that of GaAsBi, i.e., $88 \mathrm{meV} / \% .^{1}$ On the other hand, if the bandgap bowing rate is taken as $33 \mathrm{meV} / \%$ as bulk structure, ${ }^{9}$ the $\mathrm{Bi}$ content should be about $0.45 \%$, which is obviously lower than that derived by XRD and SIMS in the thin film, ${ }^{5}$ and hence a higher $\mathrm{Bi}$ exhalation has to be assumed for low dimensional structure during MBE growth process. In contrast, it seems to be very reasonable to take the value of $21 \mathrm{meV} / \%$ for the bandgap bowing of $\mathrm{GaSbBi}$, because the topmost valence-band edge position of InSb is close to that of $\mathrm{GaSb}^{33}$ and a bowing rate of about $19 \mathrm{meV} / \%$ was suggested for InSbBi. ${ }^{34}$ From this aspect, this study not only reveals the shallow-terrace-like interface structure but may also serve as an effective routine for characterizing Bi content and/or bandgap bowing effect of dilute-Bi QWs.

\section{SUMMARY}

To summarize, PL and magneto-PL measurements were conducted on two GaSbBi/AlGaSb SQW samples grown at 360 and $380^{\circ} \mathrm{C}$, and one $\mathrm{GaSb} / \mathrm{AlGaSb} \mathrm{SQW}$ sample grown at $370^{\circ} \mathrm{C}$ as reference. While redshift as foreseen by the BAC model was clearly seen in the sample grown at $380^{\circ} \mathrm{C}$, an abnormal blueshift was observed in the sample grown at $360^{\circ} \mathrm{C}$. Bimodal PL features with the similar transition origins were identified for both the GaSbBi samples by diamagnetic shift behavior. The experimental results indicate the existence of an interfacial effective terrace-like structure in the GaSbBi/AlGaSb SQW which is unidirectional to shrink the quantum well thickness other than the interfacial islands or regular bidirectional interfacial roughness. A phenomenological model is proposed to well understand the emergence of this interfacial structure based on the large-lateral-scale interface islands and the interfacial $\mathrm{Al} / \mathrm{Ga}$ interdiffusion both enhanced by $\mathrm{Bi}$ incorporation. Analysis suggests that $\mathrm{Bi}$ atoms tend to occupy the substitutional position at growth temperature of $380^{\circ} \mathrm{C}$, and $\mathrm{Bi}$ isoelectronic incorporation enhances the excitonic reduced effective mass and binding energy. A value of about $21 \mathrm{meV} / \%$ is derived for the bandgap bowing of GaSbBi. The movement of PL peak and the energy difference of bimodal features may serve as a measure for the $\mathrm{Bi}$ content and the depth of shallow terraces at interface.

\section{ACKNOWLEDGMENTS}

One of the authors (C.X.R.) thanks Xiang Lü for helpful discussion. The work was sponsored by the SITP KIP (QZY74), the STCSM (11JC1413800), and the NSFC (11274329 and 61176075) of China.

${ }^{1}$ S. Francoeur, M. Seong, A. Mascarenhas, S. Tixier, M. Adamcyk, and T. Tiedje, Appl. Phys. Lett. 82, 3874 (2003).

${ }^{2}$ S. Francoeur, S. Tixier, E. Young, T. Tiedje, and A. Mascarenhas, Phys. Rev. B 77, 085209 (2008).

${ }^{3}$ J. Yoshida, T. Kita, O. Wada, and K. Oe, Jpn. J. Appl. Phys., Part 1 42, 371 (2003).

${ }^{4}$ B. Fluegel, S. Francoeur, A. Mascarenhas, S. Tixier, E. Young, and T. Tiedje, Phys. Rev. Lett. 97, 67205 (2006).

${ }^{5}$ Y. Song, S. Wang, I. Saha Roy, P. Shi, and A. Hallen, J. Vac. Sci. Technol. B 30, 02B114 (2012).

${ }^{6}$ W. Shan, W. Walukiewicz, J. W. Ager, E. E. Haller, J. F. Geisz, D. J. Friedman, J. M. Olson, and S. R. Kurtz, Phys. Rev. Lett. 82, 1221 (1999).

${ }^{7}$ K. Alberi, J. Wu, W. Walukiewicz, K. Yu, O. Dubon, S. Watkins, C. Wang, X. Liu, Y. Cho, and J. Furdyna, Phys. Rev. B 75, 045203 (2007).

${ }^{8}$ J. Shao, W. Lu, M. Sadeghi, X. Lü, S. Wang, L. Ma, and A. Larsson, Appl. Phys. Lett. 93, 031904 (2008).

${ }^{9}$ V. Germogenov, Y. Otman, V. Chaldyshev, and Y. Shartsev, Sov. Phys. Semicond.-USSR 23, 942 (1989).

${ }^{10}$ P. Gladkov, E. Monova, and J. Weber, J. Cryst. Growth 146, 319 (1995).

${ }^{11}$ S. Das, T. Das, S. Dhar, M. de La Mare, and A. Krier, Infrared Phys. Technol. 55, 156 (2012).

${ }^{12}$ A. Mascarenhas, S. Kurtz, A. Kibbler, and J. Olson, Phys. Rev. Lett. 63, 2108 (1989).

${ }^{13}$ J. Shao, W. Lu, X. Lü, F. Yue, Z. Li, S. Guo, and J. Chu, Rev. Sci. Instrum. 77, 063104 (2006).

${ }^{14}$ X. Lu, D. Beaton, R. Lewis, T. Tiedje, and Y. Zhang, Appl. Phys. Lett. 95, 041903 (2009).

${ }^{15}$ J. Shao, L. Chen, W. Lu, X. Lü, L. Zhu, S. Guo, L. He, and J. Chu, Appl. Phys. Lett. 96, 121915 (2010).

${ }^{16}$ D. Gammon, B. Shanabrook, and D. Katzer, Phys. Rev. Lett. 67, 1547 (1991).

${ }^{17}$ A. Zrenner, L. V. Butov, M. Hagn, G. Abstreiter, G. Böhm, and G. Weimann, Phys. Rev. Lett. 72, 3382 (1994).

${ }^{18}$ K. Leosson, J. Jensen, W. Langbein, and J. Hvam, Phys. Rev. B 61, 10322 (2000).

${ }^{19}$ B. Deveaud, A. Regreny, J. Emery, and A. Chomette, J. Appl. Phys. 59, 1633 (1986).

${ }^{20}$ L. Zhu, J. Shao, X. Lü, S. Guo, and J. Chu, J. Appl. Phys. 109, 013509 (2011). 
${ }^{21}$ J. Shao, D. Haase, A. Dörnen, V. Härle, and F. Scholz, J. Appl. Phys. 87, 4303 (2000).

${ }^{22}$ J. Shao, R. Winterhoff, A. Dörnen, E. Baars, and J. Chu, Phys. Rev. B 68, 165327 (2003).

${ }^{23}$ B. Sun, D. Jiang, X. Luo, Z. Xu, Z. Pan, L. Li, and R. Wu, Appl. Phys. Lett. 76, 2862 (2000).

${ }^{24}$ Q. Zhao, S. Wang, Y. Wei, M. Sadeghi, A. Larsson, and M. Willander, Appl. Phys. Lett. 86, 121910 (2005).

${ }^{25}$ J. Shao, W. Lu, G. Tsen, S. Guo, and J. Dell, J. Appl. Phys. 112, 063512 (2012).

${ }^{26}$ S. Imhof, A. Thranhardt, A. Chernikov, M. Koch, N. Koster, K. Kolata, S. Chatterjee, S. Koch, X. Lu, S. Johnson et al., Appl. Phys. Lett. 96, 131115 (2010).
${ }^{27}$ J. Shao, A. Dörnen, R. Winterhoff, and F. Scholz, Phys. Rev. B 66, 035109 (2002).

${ }^{28}$ H. Xie, J. Katz, W. Wang, and Y. Chang, J. Appl. Phys. 71, 2844 (1992).

${ }^{29}$ C. Snyder, B. Orr, D. Kessler, and L. Sander, Phys. Rev. Lett. 66, 3032 (1991).

${ }^{30}$ Y. Song, S. Wang, I. Saha Roy, P. Shi, A. Hallen, and Z. Lai, "Molecular beam epitaxy growth of $\mathrm{InSb}_{1-\mathrm{x}} \mathrm{Bi} \mathrm{i}_{\mathrm{x}}$ thin films," J. Cryst. Growth (in press).

${ }^{31}$ G. Pettinari, A. Polimeni, J. Blokland, R. Trotta, P. Christianen, M. Capizzi, J. Maan, X. Lu, E. Young, and T. Tiedje, Phys. Rev. B 81, 235211 (2010).

${ }^{32}$ J. Fan and Y. Chen, J. Appl. Phys. 80, 1239 (1996).

${ }^{33}$ C. Van de Wall, Phys. Rev. B 39, 1871 (1989).

${ }^{34}$ J. Lee, J. Kim, and M. Razeghi, Appl. Phys. Lett. 70, 3266 (1997). 